\title{
Changes in angular size and speed affect the judged height of objects moving over a ground surface
}

\author{
Junjun Zhang and Myron L. Braunstein \\ Department of Cognitive Sciences University of California, Irvine Irvine, California, USA
}

George J Andersen

University of California, Riverside Riverside, California, USA

\begin{abstract}
Kersten et al (1997) showed that the perceived path of an object moving over a ground surface can be manipulated by changing the path of a shadow. Using a scene similar to Kersten's "ball-in-abox" scene, we investigated the effect of angular size and angular speed in determining the perceived height of a moving sphere when optical contact (the position at which the object contacted the ground in the image) indicated that the sphere was receding in depth. Four experiments examined both the effects of changes in size and speed and the effects of constant levels of size and speed. Increases in angular size or speed during a motion sequence resulted in judgments of increased height above the ground plane. The angular size at the end of the motion sequence was also important in determining judged height, with greater height judged with larger final sizes.
\end{abstract}

\section{Introduction}

According to Gibson's (1950) "ground theory," a major factor in determining the perceived location of an object in a 3D scene is optical contact - the position on the image of the ground surface that is in contact with the image of the object. There is considerable evidence supporting Gibson's observation (Loomis et al 1996; Sinai et al 1998; Wu et al 2004). The importance of ground contact in determining the perceived location of objects generalizes to scenes in which objects are resting on other objects, which in turn are resting on the ground, giving rise to nested contact relations (Meng and Sedgwick 2001, 2002).

As Gibson also observed, other variables can affect the degree to which perceived distance along a ground surface is determined by optical contact. Kersten et al (1997) showed that an object will be perceived as rising above the ground, rather than moving along the ground, when the motion of a shadow is consistent with the object increasing in elevation. Ni et al $(2004,2005,2007)$ examined the effects of combining information from optical contact with shadow, motion parallax and occlusion on the perceived location of an object in a scene. Perceived height was not measured directly, but geometrically an object elevated above the ground would be closer than the location indicated by optical contact. The similar effects of shadow on judged height (Kersten et al 1997) and on judged location in depth (Ni et al 2004) suggest that observers perceive this relation between height and location.

If an object is moving relative to a ground surface that is receding in depth, and there is no additional information about its distance (e.g. relative motion parallax, binocular disparity, 
occlusion or shadow), there are three variables that may be expected to affect the perceived distance of the object: optical contact, changes in angular size and changes in angular speed. Consider displays in which a texture gradient on the ground surface is consistent with a surface receding in depth and optical contact indicates that the object is moving from the near edge to the far edge of the surface. There are three perceptual constraints or assumptions that are relevant to the perception of the position of the object in the scene: (1) An otherwise unsupported object in a 3D scene is in contact with the ground surface. (2) An object does not change its size while moving in a 3D scene. (3) An object maintains a constant velocity in 3D while moving in a scene. The perceived changes in location of an object could be consistent with all three constraints if the angular size and angular speed of the object decreased consistently as its optical contact indicated recession in depth, that is, as the object's image contacted the image of the ground surface at increasing distances. Optical contact and angular size and speed information would also be consistent if angular size and speed both increased as optical contact indicated that the object was approaching. If, on the other hand, angular size increased as optical contact indicated increasing distance, then a perception should result that relaxes one or both of the relevant constraints: The object could be perceived as rising above the ground or changing its 3D size or both. Similarly, an object may be perceived as rising above the ground or changing its speed in 3D or both if the angular speed increased as optical contact indicated that the object was receding in depth.

Changes in angular size and angular speed during object motion are sufficient for determining motion direction in 3D (Todd 1981; Regan and Kaushal 1994) and for determining the curvature of the trajectory of a moving object (Zhang et al 2011). Size and speed effects also have been studied in the context of collision detection (e.g. Andersen et al 1999). Kersten et al (1997) compared the effects of changes in angular size consistent with the ball remaining at the bottom of the box to a constant angular size, combined with corresponding or consistent shadow information. Angular size affected judgments for some observers, but shadow was the dominant variable. Shadow effects are likely to dominate both size and speed effects and the purpose of the present study was to examine size and speed effects separately.

In the present study, we examined the effect of changes in angular size and angular speed on the perceived height of a sphere in displays of a sphere moving over a ground surface. In the first experiment, we varied the relation between optical contact and angular size, with the angular size of the object either decreasing, remaining constant or increasing as optical contact indicated that the object was receding in depth. The relation between optical contact and angular speed was varied in a similar manner in the second experiment. In the third experiment, we varied both the relations between optical contact and angular size and between optical contact and angular speed, keeping the final angular size and speed constant. The fourth experiment examined the effect of constant levels of size and speed. In all experiments, the dependent measure was a judgment of the height at which the object would impact a vertical plane if its motion continued.

\section{Experiment 1: Variations in angular size}

This experiment examined the effect of variations in the angular size of a sphere on judgments of the object's height, with the angular speed of the sphere held constant.

\subsection{Method}

2.1.1 Stimuli-The displays used a scene similar to that used by Kersten et al (1997), consisting of a textured ground surface with vertical sidepieces on the left and right. The ground surface was covered with red and white squares and the sidepieces were covered with red and white horizontal stripes. The major difference between our scene and Kersten 
et al's "ball-in-a-box" scene is that we added a platform in the lower left corner of the box (see Figure 1). The platform was added after pilot studies indicated that it was difficult for observers to perceive the sphere as being above the ground at any time during the display if it was resting on the ground at the beginning of the motion sequence. The red and white squares on the ground surface were randomly rearranged for each trial.

In the 3D simulation, the ground surface was $73.7 \mathrm{~cm}$ wide $\times 294.8 \mathrm{~cm}$ deep, the platform was $18.4 \mathrm{~cm}$ wide $\times 36.8 \mathrm{~cm}$ deep $\times 4.6 \mathrm{~cm}$ high, the height of the sidepieces was $18.4 \mathrm{~cm}$ and the eyepoint was $183 \mathrm{~cm}$ from the near edge of the ground surface. On the monitor screen, the top edge of the textured ground surface was $28.2 \mathrm{~cm}\left(8.8^{\circ}\right)$ wide, the bottom edge was $73.7 \mathrm{~cm}\left(23^{\circ}\right)$ wide and the vertical separation between the top and bottom edges was $14.4 \mathrm{~cm}\left(4.5^{\circ}\right)$. The top edge of the platform was $18.4 \mathrm{~cm}\left(5.8^{\circ}\right)$ wide, the bottom edge was $15.3 \mathrm{~cm}\left(4.8^{\circ}\right)$ wide and the vertical separation between the edges was $3.1 \mathrm{~cm}\left(0.97^{\circ}\right)$. The near edges of the sidepieces were $18.4 \mathrm{~cm}\left(5.8^{\circ}\right)$ high, the far edges were $7.0 \mathrm{~cm}\left(2.2^{\circ}\right)$ high and the horizontal separation between the two edges was $22.5 \mathrm{~cm}\left(7.0^{\circ}\right)$.

Each display began with a stationary sphere on the platform. A sphere was used because it does not change its projected shape with changes in orientation. After 500 milliseconds, the sphere moved toward the upper right of the ground surface and disappeared before it reached the right sidepiece. The sphere's path in the image was tilted $12.1^{\circ}$ relative to the horizontal and was identical in each display.

2.1.2 Observers-The observers were 12 undergraduates at the University of California, Irvine who received extra credit for participating. All observers had visual acuity (corrected if required) of at least 20/40 (Snellen Eye Chart) and were naïve about the purpose of the experiment.

2.1.3 Apparatus and procedure-The displays were computer-generated and presented on a 46-inch $(117 \mathrm{~cm})$ LCD monitor at a resolution of $1920 \times 1080$ with a refresh rate of 60 Hz. Observers viewed the displays monocularly through a viewing tube in a darkened room. The eye to screen distance was $183 \mathrm{~cm}$. The eye position was aligned vertically with a point $23.3 \mathrm{~cm}\left(7.3^{\circ}\right)$ above the lower edge of the textured ground surface and the eye position was aligned horizontally with the center of the ground surface. Black cloth was placed around the monitor and viewing tube to assure that the monitor was visible only through the viewing tube. The viewing tube limited the field of view to an area within the monitor. The frame of the monitor was not visible. The observers were told to imagine that after the "ball" disappeared, it would keep going and hit the right sidepiece. The task was to judge the height at which the center of the sphere would hit the sidepiece. After the sphere disappeared, a horizontal blue line appeared on the right sidepiece, centered vertically 9.2 $\mathrm{cm}$ above the ground plane. The observers indicated their height judgments by adjusting the height of the blue line with a joystick.

2.1.4 Design-There were two independent variables: the angular size of the sphere at the start of the motion sequence and the angular size at the end of the sequence. The angular size at the start and at the end of the motion had the same three levels: $0.44^{\circ}$ ("small"), $0.82^{\circ}$ ("medium") and $1.52^{\circ}$ ("large"). The corresponding diameters on the monitor screen were $1.42 \mathrm{~cm} 2.62 \mathrm{~cm}$ and $4.88 \mathrm{~cm}$. The angular size conditions simulated three types of motion in 3D space. Constant angular size (e.g., starting with a small size and ending with a small size) simulated motion of the sphere in a frontal plane. Decreasing angular size (e.g., starting with a medium size and ending with a small size) simulated the sphere receding in depth on the ground plane. Increasing angular size (e.g., starting with a small size and ending with a medium size) was the reverse of the decreasing angular size function and simulated approach of the sphere in 3D space. The change in angular size on each trial was linear 
across the motion path. The angular speed of the sphere was $4 \% \mathrm{~s}(12.9 \mathrm{~cm} / \mathrm{s})$ in all conditions. There were 3 blocks of 45 trials, with a two-minute rest period between blocks. In each block, there were 5 trials for each condition. The first block was a practice block and was not used in the data analysis.

\subsection{Results and discussion}

The observer's task was to judge the height at which the center of the sphere would hit the right sidepiece. Because the radius of the sphere varied across conditions, the radius was subtracted from the judged height so that the dependent measure would reflect the position of the bottom of the sphere. (Alternatively, we could have asked for judgments based on the height of the bottom of the sphere, but we believed that these would be more difficult for observers.) The adjusted judged heights are show in Figure 2.

An ANOVA showed significant main effects for start size, $F_{2,22}=4.96, p<0.05$, and end size, $F_{2,22}=18.6, p<0.01$. The interaction was not significant, $F_{4,44}=2.06, p>0.05$. Judged height was lower when the start size was large than when the start size was small ( $p$ $<0.05$ ) or medium $(p<0.05)$. (All pairwise comparison used the least significant differences (LSD) test.) The judged heights with the small, medium and large start sizes were $3.77 \mathrm{~cm}, 3.46 \mathrm{~cm}$ and $2.80 \mathrm{~cm}$, respectively. Judged height was greater with a large end size than with a medium end size $(p<0.01)$, and was greater with a medium end size than with a small end size $(p<0.01)$. The judged heights with the small, medium and large end sizes were $1.80 \mathrm{~cm}, 3.11 \mathrm{~cm}$ and $5.12 \mathrm{~cm}$, respectively.

Because we were primarily interested in the effects of the change in angular size on height judgments, we reorganized the nine size change conditions into three categories: (1) decreasing angular size, consistent with recession in depth (medium to small, large to medium and large to small), (2) constant angular size, consistent with constant depth (small to small, medium to medium and large to large) and (3) increasing angular size, consistent with approach in depth (small to medium, small to large and medium to large). An ANOVA for the reorganized data showed a significant main effect for the type of size change, $F_{2,22}=$ $12.7, p<0.01$. Judged height was greater with increasing angular size than with constant angular size $(p<0.01)$, and was greater with constant angular size than with decreasing angular size $(p<0.01)$. The judged heights with decreasing, constant and increasing sizes were $1.90 \mathrm{~cm}, 3.28 \mathrm{~cm}$ and $4.85 \mathrm{~cm}$, respectively. This result is consistent with the expectation that when optical contact indicates recession in depth but angular size change does not indicate recession in depth, the perceived height of the object should increase.

\section{Experiment 2: Variations in angular speed}

This experiment examined the effect of variations in angular speed on judgments of the height at which the sphere would contact the sidepiece, with the angular size of the sphere held constant.

\subsection{Method}

The method was similar to that used in Experiment 1, except that angular speed change was manipulated with the angular size of the sphere held constant during the motion. An additional twelve naïve observers from the same population participated. The visual acuity requirement was the same as in Experiment 1.

3.1.1 Design-The design was similar to that in Experiment 1, except that the independent variables were the start speed and end speed. There were three levels for both the start and end angular speed: $1.2 \%$ ("slow"), $4.0 \%$ s ("medium") and $13.8 \%$ ("fast"), resulting in nine 
conditions for the speed change. The speeds on the monitor corresponding to the three angular speeds were $3.7 \mathrm{~cm} / \mathrm{s}, 12.9 \mathrm{~cm} / \mathrm{s}$ and $44.3 \mathrm{~cm} / \mathrm{s}$. The angular speed conditions simulated the same three types of motion in 3D space as the angular size conditions, with constant angular speed simulating motion in a frontal plane, decreasing angular speed simulating motion in depth along the ground plane and increasing angular speed simulating approach in depth, the reverse of the receding motion. The change in angular speed on each trial was linear across the motion path. The diameter of the sphere on the screen was 1.84 $\mathrm{cm}\left(0.58^{\circ}\right)$ in all conditions. There were 3 blocks of 45 trials, with a two-minute rest period between blocks. In each block, there were 5 trials for each condition. The first block was a practice block and was not used in the data analysis.

\subsection{Results and discussion}

The dependent measure was the height at which the observer positioned the horizontal marker on the right sidepiece, adjusted by subtracting the radius of the sphere. (The radius did not vary across conditions in this experiment, but the adjustment was made in all experiments for consistency in reporting the results.) The adjusted judged heights are shown in Figure 3.

An ANOVA found a significant main effect for end speed, $F_{2,22}=5.92, p<0.05$. Judged height with the fast end speed was greater than with either the slow $(p<0.05)$ or medium $(p$ $<0.01)$ end speeds. The judged heights with the slow, medium and fast end speeds were $3.95 \mathrm{~cm}, 4.35 \mathrm{~cm}$ and $4.73 \mathrm{~cm}$, respectively. The main effect of start speed, $F_{2,22}=0.423, p$ $>0.05$ and the interaction, $F_{4,44}=0.629, p>0.05$, were not significant. To study the effect of speed change, the data were reorganized into three categories based on the relation between the start and end speeds: decreasing (fast to slow, fast to medium and medium to slow), constant (slow to slow, medium to medium and fast to fast) and increasing (slow to medium, medium to fast, and slow to fast). An ANOVA found a significant main effect of speed change, $F_{2,22}=6.78, p<0.05$. Judged height was lower with decreasing speed than with either constant $(p<0.01)$ or increasing $(p<0.05)$ speed. The judged heights with decreasing, constant and increasing speed were $4.03 \mathrm{~cm}, 4.43 \mathrm{~cm}$ and $4.58 \mathrm{~cm}$, respectively.

\section{Experiment 3: Variations in size and speed}

A limitation in examining the results of Experiment 1 according to whether the angular size was decreasing, remaining constant or increasing, is that the range of end sizes varied across these categories. For example, the decreasing size category could include only small and medium end sizes. Similarly, in Experiment 2 the end speeds varied across decreasing, constant and increasing speed conditions. In order to examine the effects of changes in angular size and angular speed on judged height independently of end size and speed, we used the same end size and end speed in all conditions in the present experiment. Also, with the directions of size and speed changes varied independently, this experiment can provide an indication of the relative importance of these two types of changes in determining judged height.

\subsection{Method}

The stimuli were similar to those in the previous experiments. Both angular size and angular speed were manipulated. The three levels of start size were the same as in Experiment 1 and the three levels of start speed were the same as in Experiment 2. Both the end angular size and the end angular speed were medium on all trials. Table 1 shows the start size and speed for each of the nine conditions, the changes in size and speed and the relative changes in the two variables, to be discussed in the Results section. Both the changes in angular size and angular speed were linear across the motion path. An additional 16 observers participated in 
this experiment. They were from the same population and met the same visual acuity requirement. There were 3 blocks of 45 trials, with a two-minute rest period between blocks. In each block, there were 5 trials for each condition. The first block was a practice block and was not used in the data analysis.

\subsection{Results and discussion}

The results are shown in Figure 4. An ANOVA showed significant main effects for both size change, $F_{2,30}=6.85, p<0.05$, and speed change, $F_{2,30}=5.37, p<0.05$. The interaction was not significant, $F_{4,60}=0.631, p<0.05$. Pairwise comparisons showed that judged height was greater with increasing angular size than with constant angular size $(p<0.05)$, and was greater with constant angular size than with decreasing angular size $(p<0.05)$. The mean judged heights with decreasing, constant and increasing size were $3.29 \mathrm{~cm}, 4.00 \mathrm{~cm}$ and $4.62 \mathrm{~cm}$, respectively. Judged height was greater with increasing angular speed than with decreasing $(p<0.05)$ or constant $(p<0.01)$ angular speed. The mean judged height with decreasing, constant and increasing speeds were $3.82 \mathrm{~cm}, 3.87 \mathrm{~cm}$ and $4.23 \mathrm{~cm}$, respectively. These results indicate that, even with the end size and speed identical in each condition, changes in angular size and speed can affect the judged height of the sphere.

With both changes in size and speed manipulated, these two types of information could be consistent or in conflict with each other. For three conditions, changes in size and speed were consistent: both increasing, both constant, or both decreasing. These conditions are designated "same" in the rightmost column of Table 1. An ANOVA on these three consistent conditions showed a significant main effect, $F_{2,30}=7.01, p<0.01$, with judged height greater with increasing size and speed than with either decreasing $(p<0.05)$ or constant ( $p<0.01)$ size and speed. The mean judged heights with decreasing, constant and increasing size and speed were $3.26 \mathrm{~cm}, 3.88 \mathrm{~cm}$ and $4.91 \mathrm{~cm}$, respectively. The six conflicting conditions were divided into two groups: (1) displays with size increasing relative to speed (size increasing with speed decreasing, size increasing with speed constant or size constant with speed decreasing) and (2) speed increasing relative to size (speed increasing with size decreasing, speed increasing with size constant or speed constant with size decreasing). A t-test showed a significant main effect, $t_{15}=2.18, p<0.05$. Judged height was greater for the conditions with size increasing relative to speed than for the conditions with speed increasing relative to size. The mean judged heights for the conditions with size increasing relative to speed and the conditions with speed increasing relative to size were $4.25 \mathrm{~cm}$ and $3.64 \mathrm{~cm}$, respectively. These results suggest that, although both size and speed affect the judged height, when these two types of information conflict the effect of size overrides the effect of speed.

\section{Experiment 4: Constant size and speed}

In the first two experiments, we found that both the end size and end speed were important in determining the perceived height of the sphere. It is possible that a sphere having a greater angular size or moving at a greater angular speed would be perceived as closer, thus inducing a perception of greater height. In Experiment 1 and 2, the effect of end size and speed were not studied separately from the effects of changes in size and speed. In the present experiment, both size and speed were constant during a display. We wanted to determine whether a constant larger size or a constant faster speed would induce a judgment of greater height.

\subsection{Method}

The stimuli were similar to those in the first two experiments except that the angular size and speed of the sphere were held constant during a trial. The three levels of size were the 
same as in Experiment 1 and the three levels of speed were the same as in Experiment 2, resulting in nine speed-size combinations. There were 3 blocks of 45 trials with a 2-minutes rest between blocks. In each block, there were 5 trials for each condition. The first block was for practice and the results were not used in the data analysis. An additional twelve observers from the same population participated. They met the same visual acuity requirement.

\subsection{Results and discussion}

The adjusted judged height in each condition is shown in Figure 5. An ANOVA found a significant main effect for size, $F_{2,22}=24.5, p<0.01$. The main effect of speed, $F_{2,22}=$ $2.59, p>0.05$, and the interaction, $F_{4,44}=2.00, p>0.05$, were not significant. Judged height was greater with the large angular size than with the medium size $(p<0.01)$, and was greater with the medium size than with small size $(p<0.01)$. The mean judged heights with the small, medium and large constant sizes were $1.87 \mathrm{~cm}, 2.88 \mathrm{~cm}$ and $4.57 \mathrm{~cm}$, respectively.

\section{Summary and conclusion}

We examined the effects of changes in the angular size and speed of a moving object on the perceived height of the object, with optical contact with a ground surface indicating that the object was receding in depth. The effects of changes in angular size and speed were examined separately in Experiments 1 and 2. Judged height was greater with increasing angular size than with constant angular size, and was greater with constant angular size than with decreasing angular size. Judged height was greater with either constant or increasing speed than with decreasing speed. The angular size and speed at the end of the motion sequence also affected judged height. Judged height was greater with a large end size than with a medium end size and greater with a medium end size than with a small end size. Judged height was greater with a faster end speed than with either a slow or medium end speed. The results of Experiments 1 and 2 thus indicate that either changes in angular size and speed or the final angular size and speed in the motion sequence can affect the perceived height of an object. These two factors could not be studied separately in Experiments 1 and 2, so Experiments 3 and 4 were conducted to study the effect of changes in size and speed and the effect of final size and speed, respectively.

In Experiment 3 we studied the effect of changes in size and speed on the perceived height, while keeping constant any effect of final size or speed. Judged height was greater with increasing angular size than with constant angular size, and was greater with constant angular size than with decreasing angular size. Judged height was greater with increasing angular speed than with decreasing or constant angular speed. For conditions in which the direction of change in size was not the same as the direction of change in speed, the size change information appeared to dominate.

Experiment 4 examined the effect of different levels of constant size and speed on judged height. Judged height was greater with a large angular size than with a medium angular size, and was greater with a medium angular size than with a small angular size. Previous research indicates that distance judgments can be affected by prior knowledge of the size of familiar objects (Gogel and Da Silva 1987; Predebon 1992). Distance is overestimated for objects reduced in size and is underestimated for enlarged object. In Experiment 4, although the actual size of the sphere was not known to the observers, they may have assumed that its 3D size was the same on all trials. Thus, the smaller size may have suggested a greater distance and the larger size may have suggested a lesser distance. The perception of an object at a greater distance than that indicated by optical contact is likely to result in the object being perceived at a greater height. As noted in the Introduction, this implication is 
consistent with the geometry and is supported by studies showing similar effects with similar stimulus manipulations, with either judged height (Kersten et al 1997) or judged distance (Ni et al 2004) as the dependent measure.

Although judged height was affected by changes in angular size and speed, by the final angular size and speed and by constant levels of angular size and speed, the magnitude of these effects was not large. This is indicative of the dominance of the optical contact constraint. It is difficult for observers to perceive an object in optical contact with a ground surface as rising above the ground. In pilot studies, before we introduced an elevated platform in the starting position, the pattern of effects was similar but the magnitudes were smaller. A comparison of our overall findings to those of Kersten et al (1997) and Ni et al (2004) suggests that the constraint that objects in optical contact with a ground surface are located on the ground is more easily overcome by conflicting shadow information than by conflicting size change and speed change information. Our results also indicate that a constant 3D size constraint is more important than a constant 3D speed constraint. This is not surprising because 3D objects do not usually change in size but can change in 3D speed while in motion.

In the present study, the projected motion of the sphere was always upward, so that the angular declination (the angle between a horizontal line at eye level and a line from the eye to the object) always decreased. Decreasing angular declination indicates recession in depth (Ooi et al 2001, 2006) and would have provided information corresponding to the information provided by optical contact.

In summary, we found that with optical contact indicating recession in depth, increases in angular size or speed are attributed, at least in part, to increases in height above the ground plane. The angular size and speed of the moving object, either throughout the motion sequence or at the end of the sequence, were also important in determining judged height at the end of the motion sequence. Overall, we can conclude that angular size and angular speed can alter the perceived height of objects moving across a ground surface, with angular size the more important variable.

\section{Acknowledgments}

We would like to thank Brianna Phelps and Amy Vu for their assistance with this research. This project was supported by a grant from the National Institutes of Health (EY18334) to GJA.

\section{References}

Andersen GJ, Cisneros J, Atchley P, Saidpour A. Speed, size, and edge-rate information for the detection of collision events. Journal of Experimental Psychology: Human Perception and Performance. 1999; 25:256-269. [PubMed: 10069034]

Gibson, JJ. The perception of the visual world Boston. Houghton-Mifflin; 1950.

Gogel WC, Da Silva JA. Familiar size and the theory of off-sized perceptions. Perception \& Psychophysics. 1987; 41:318-328. [PubMed: 3588229]

Kersten DP, Mamassian P, Knill DC. Moving cast shadows induce apparent motion in depth. Perception. 1997; 26:171-192. [PubMed: 9274752]

Loomis JM, DaSilva JA, Philbeck JW, Fukusima SS. Visual perception of location and distance. Current Directions in Psychological Science. 1996; 5:72-77.

Madison C, Thompson W, Kersten D, Shirley P, Smits B. Use of interreflection and shadow for surface contact. Perception \& Psychophysics. 2001; 63:187-194. [PubMed: 11281095]

Meng JC, Sedgwick HA. Distance perception mediated through nested contact relations among surfaces. Perception \& Psychophysics. 2001; 63:1-15. [PubMed: 11304007] 
Meng JC, Sedgwick HA. Distance perception across spatial discontinuities. Perception \& Psychophysics. 2002; 64:1-14. [PubMed: 11916293]

Ni R, Braunstein ML, Andersen GJ. Perception of scene layout from optical contact, shadows, and motion. Perception. 2004; 33:1305-1318. [PubMed: 15693673]

Ni R, Braunstein ML, Andersen GJ. Distance perception from motion parallax and ground contact. Visual Cognition. 2005; 12:1235-1254.

Ni R, Braunstein ML, Andersen GJ. Scene layout from ground contact, occlusion, and motion parallax. Visual Cognition. 2007; 15:48-68.

Ooi TL, Wu B, He ZJ. Distance determined by the angular declination below the horizon. Nature. 2001; 414:197-200. [PubMed: 11700556]

Ooi TL, Wu B, He ZJ. Perceptual space in the dark affected by the intrinsic bias of the visual system. Perception. 2006; 35:605-624. [PubMed: 16836053]

Predebon J. The role of instructions and familiar size in absolute judgments of size and distance. Perception \& Psychophysics. 1992; 51:344-354. [PubMed: 1603648]

Regan D, Kaushal S. Monocular discrimination of the direction of motion in depth. Vision Research. 1994; 34:163-177. [PubMed: 8116276]

Sinai MJ, Ooi TL, He ZJ. Terrain influences the accurate judgement of distance. Nature. 1998; 395:497-500. [PubMed: 9774104]

Todd JT. Visual information about moving objects. Journal of Experimental Psychology: Human Perception and Performance. 1981; 7:975-810. [PubMed: 6457104]

Wu B, Ooi TL, He ZJ. Perceiving distance accurately by a directional process of integrating ground information. Nature. 2004; 428:73-77. [PubMed: 14999282]

Zhang J, Braunstein ML, Andersen GJ. The effects of size and speed on perceived 3D object motion at different distances. Journal of Vision. 2011; 11:738. 


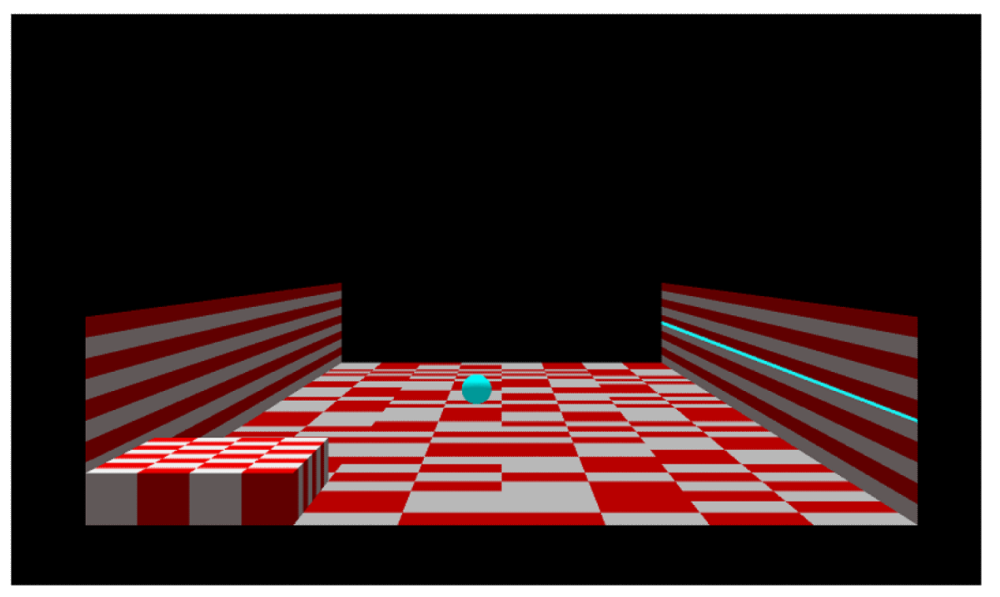

Figure 1.

A frame from the motion sequence. The sphere moved from lower left to upper right. Observers adjusted a horizontal marker on the right sidepiece to indicate the height at which the ball would hit the sidepiece. In the actual displays the marker did not appear until the sphere completed its motion and disappeared. The thickness and brightness of the marker were increased for this illustration. 


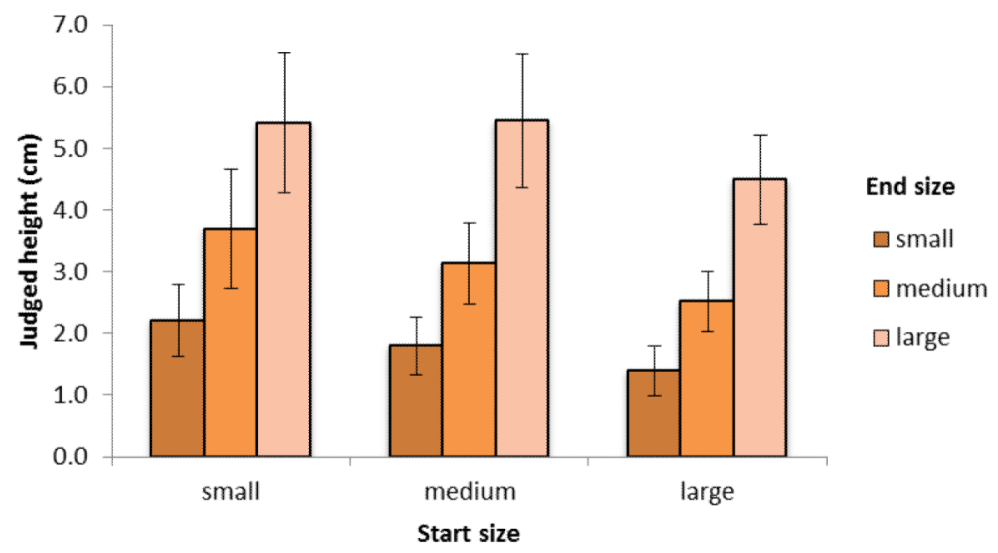

Figure 2.

Judged height as a function of variations in the angular size of the sphere (Experiment 1). 


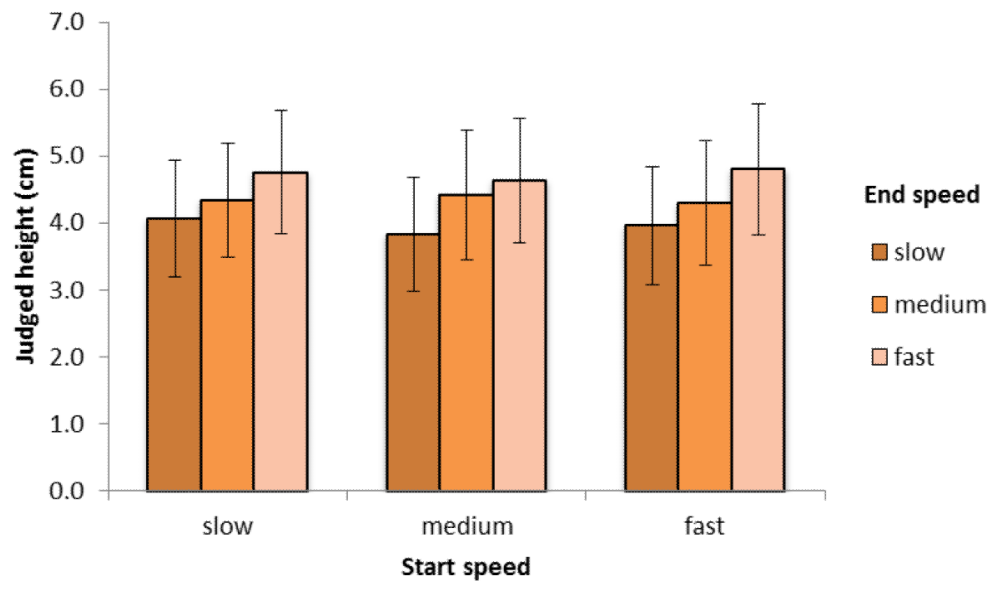

Figure 3.

Judged height as a function of variations in the angular speed of the sphere (Experiment 2). 


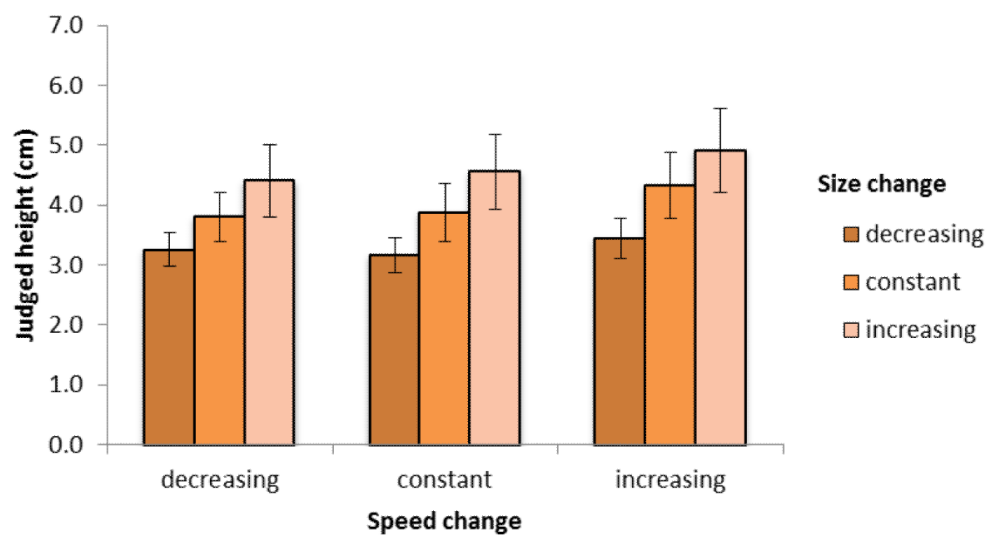

Figure 4.

Judged height as a function of variations in both the size and speed of the sphere (Experiment 3). 


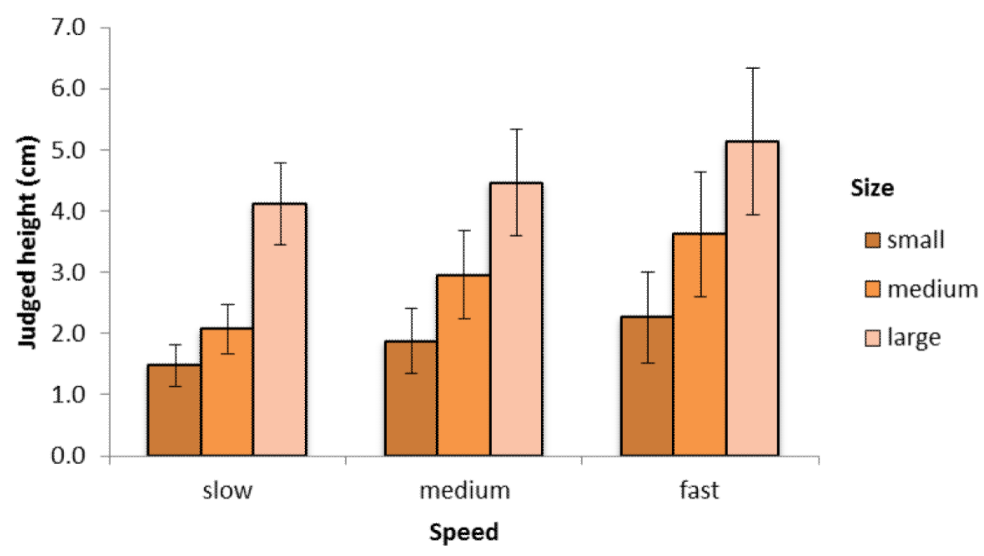

Figure 5.

Judged height as a function of constant levels of size and speed (Experiment 4). 
Table 1

The nine stimulus conditions in Experiment 3

\begin{tabular}{ccccc}
\hline start size & start speed & size change & speed change & $\begin{array}{c}\text { variable with greater relative } \\
\text { increase }\end{array}$ \\
\hline small & slow & increasing & increasing & same \\
small & medium & increasing & constant & size \\
small & fast & increasing & decreasing & size \\
medium & slow & constant & increasing & speed \\
medium & medium & constant & constant & same \\
medium & fast & constant & decreasing & size \\
large & slow & decreasing & increasing & speed \\
large & medium & decreasing & constant & speed \\
large & fast & decreasing & decreasing & same \\
\hline
\end{tabular}

Perception. Author manuscript; available in PMC 2013 November 11. 